\title{
From Game Events to Team Tactics: Visual Analysis of Dangerous Situations in Multi-Match Data
}

\author{
Manuel Stein ${ }^{1}$, Halldór Janetzko ${ }^{1}$, Andreas Lamprecht ${ }^{1}$, Daniel Seebacher ${ }^{1}$, Tobias Schreck ${ }^{2}$, Daniel Keim ${ }^{1}$, and Michael Grossniklaus ${ }^{1}$ \\ ${ }^{1}$ Department of Computer and Information Science, University of Konstanz \\ Email: firstname.lastname@uni.kn \\ ${ }^{2}$ Institute for Computer Graphics and Knowledge Visualization, Graz University of Technology \\ Email: first. lastname@cgv.tugraz.at
}

\begin{abstract}
Sport analytics in general and soccer analytics in particular constitute quickly growing markets when it comes to professional analyses and visualizations. From a data analysis research perspective, soccer is a rich source of geospatial and temporal movement data, with high details and a controlled environment. However, soccer movement is complex as its compounds are actions and reactions of two opposing teams with inverse goals. Common analyses performed today are typically oriented towards statistical analysis and considering aggregate measurements. In this work, we propose a set of effective visual-interactive methods for investigating set plays as a first step towards semi-automated analysis of tactic behavior. In our analytic design, we follow the so-called Informationseeking Mantra by Ben Shneiderman by providing overview visualizations, interactive refinements, and detailed analysis views. We take an applied approach in showing case studies that give evidence for the applicability and merits of our proposed techniques.
\end{abstract}

\section{Introduction}

Soccer, being the world's most popular invasive team sport, can be seen as a billion Euro business with many clubs, fan base, and supporting industries. Sponsors and shareholders invest substantial funds to ensure the competitiveness of their teams. Both expectations for high performance, as well as the pressure to succeed, grow steadily. Consequently, nothing is left to chance and scientific methods are introduced from fields including biomedical analysis, business operation research, but also data analysis. Specialists in several fields work jointly to create environments to optimize sport team performance. Over decades, soccer matches were manually processed, making use of match segments in video analysis. These analysts identify, extract and summarize interesting and match deciding scenes in highlight videos. This approach is very time consuming and depends highly on the chosen scenes. Furthermore, the analysis depends on the interpretations by the analysis staff, which may be subjective and difficult to reproduce in many cases. Recent technical progress in GPS-, sensor- and camera-based tracking enables the collection of large amounts of data with high temporal and spatial accuracy. The large amount of raw soccer data is often used only partially in current practice when investigating soccer matches [1].

The eventual result of a soccer match are the final scores. However, the result does not have to be positively correlated with the performance of individual players on the pitch, or constructs such as team strategy and its adaption during a match. Player behavior is subject to rules that need to be followed and the match plan of the coach. The match plan can be defined as how the coach wants his team to act. Usually, a team defends their half when they are not in possession of the ball against the opposing team and attack when they are in possession of the ball. The movement of each player depends on the movement of all other players. These strong interdependencies result in movement patterns where every action causes a reaction [2]. Cooperative behavior within teams and competitive behavior between teams are the basic ingredients of the observed movement.

As the quantity and complexity of recorded movement data and application questions in soccer steadily increases, neither purely visual nor purely analytic approaches are sufficient. Applying automated data mining techniques without any knowledge of the data will lead to incomplete and maybe even incorrect analysis results. Visualizing the data first (visual overview) provides the opportunity to use the human perception and cognition interpreting the data as well as human pattern recognition identifying relations and interdependencies. Consequently, the combination of analysis, visualization, and human domain knowledge is a promising approach for a successful in-depth analysis. Visual Analytics [3] is a core-technology postulating the synergy of machine (fast and accurate computations) and human (creativity and world knowledge). The key interfaces between humans and machines are information visualization and human computer interaction.

Ben Shneiderman, one of the founders of the field of Information Visualization, introduced an utmost influential analysis paradigm relying on interactive visualization. Specifically, the so-called visual information-seeking mantra suggests to "overview first, zoom and filter, then detail-ondemand" [4]. Following this paradigm results in overview visualizations allowing interactive zooming and filtering capabilities on interesting aspects of the data. The last step is a detailed view or analysis to verify hypothesis 


\section{Data aggregation enabling overview}

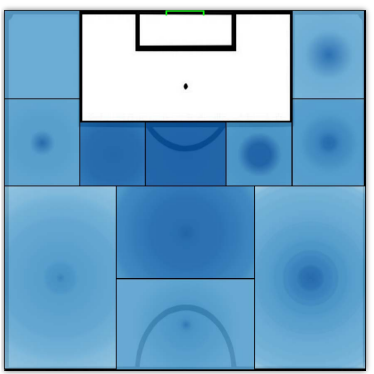

(a)

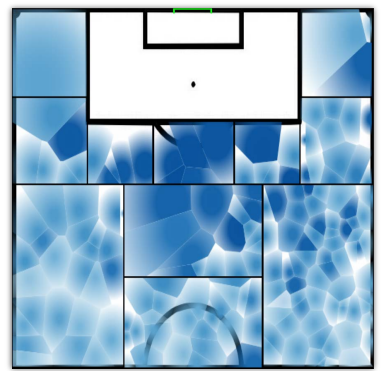

(b)

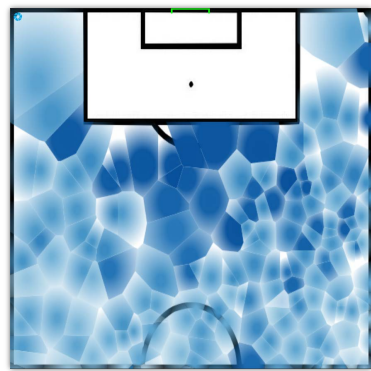

(c)

\section{Detailed representation of observations}

Figure 1. Different Levels Of Aggregation.

and gain insights. In this paper, we follow the visual information-seeking mantra and propose for the different steps in the process appropriate analysis and visualization methods supporting the analysis of set play in soccer.

We contribute methods addressing the problem of identifying a team's match plan. A team usually plays with a certain formation, depending on many influence factors such as the match situation or the current score. Consequently, there exists a myriad of possible strategic approaches, tactics, observable behavior patterns and many more parameters that need to be analyzed and which make the analysis difficult. As a first approximation to the difficult high-level task of understanding and analyzing match strategies, we focus on the analysis of match situations for which most of the parameters are known. Especially set play situations such as free kicks or corner kicks represent interesting examples of such analyses as they are executed over and over again during training. Since up to one third of all goals are scored after a set play [5], they have a significant influence on the failure or success of a team. Additionally, during a set play the match is paused which allows each player to adopt her or his position based on the tactic of the coach. We develop and integrate novel visualization techniques and allow analysts to inspect the tactic of a team during set plays.

\section{Background and Related Work}

Data-driven sport analytics has recently seen large interest both from research and practice. Especially, team sports offer a diverse variety of aspects challenging to analyze, ranging from video analysis, over movement analysis to the analysis of collective and tactical team behavior. Data-driven sports analytics has benefited to a large extent from the availability of data, which nowadays can be captured mostly automatically. For example, movement data can nowadays be captured using Computer Vision approaches (from video input) or position sensing (using triangulation).

Existing sports analytics methods can be distinguished by the type of analysis performed. Statistical data analysis relies on forming descriptive statistics from data (e.g., performance statistics) and evaluation of models (e.g., prediction of outcome of matches). Statistical approaches can be assisted by data visualization to represent the outcome of the analysis in form of graphs. For example, several systems were proposed with the goal of breaking down and simplifying a single match (or set of matches). To represent the course of a game, Legg et al. [6] use a timeline to visualize important events such as shot on target and additional information in an abstracting and summarizing manner. Space is an important concept in assessing team sports and various computational models exist to estimate space and its usage. For example, Voronoi cells have been used to this end [7].

Explorative analysis tools often do not base on particular predefined statistical models, but allow more explorative analyses, providing tools to inspect data from different perspectives. Such approaches may be combined with descriptive statistic approaches, once appropriate models are found by explorative analysis. Interactive data visualization provides many tools to support explorative analysis in sports, as has been recently identified [8]. An example of a visualexplorative system is Perin et al. [9], grouping different aspects of a soccer match such as executed passes or phases of a match and contrast them with each other in a smallmultiple view. Horton et al. [10] classify executed passes during a soccer match and evaluate the quality of these passes by means of distance, shot strength and oppression of the alluded recipient. In our own previous work, we have relied extensively on interactive visualization to explore soccer data. We proposed a multi-view analysis of derived soccer features such as player speed, acceleration, and shots on 
goal in order to segment interesting sequences from the matches for detailed inspection [11]. We considered the derived notion of free space during the match and provided a dynamic visualization that allows the use of free space by the teams in various game situations to be assessed [12] Free space is also an example of a measure that can be derived from the movement data in combination with certain assumptions (model). Specifically, attributes such as speed and viewing direction of a player should be considered when estimating free spaces that could be occupied by other team players in a given situation.

In team sports in general, the rules are fixed and the participants are trained to follow these rules, and actionreaction dynamics are set to happen. Perl et al. [13] introduce an approach to the analysis of tactical performance, where a combination of neural network-based pattern analysis and analysis of statistics such as ball possession is used. Especially the collective behavior in terms of movement is interesting, where the spatial-temporal positioning data of the players is analyzed. With the help of player movement in a match, Gyarmati et al. [14] deduce pass strategies that are indicative of prolonged ball possession. Bartlett et al. [15] in contrast explore offensive strategies across several matches with the goal of assigning roles to the individual players.

Many of the techniques developed for soccer may be applied to other team sports, such as Rugby [16] and Ice Hockey [17]. But also for individual-oriented sports, domainspecific visualization have been proposed to date [18] and the field of visual sport analytics continues to thrive.

\section{Overview}

The common first step of exploring and investigating an unknown data set is to examine the overall data structure and distribution from an overview perspective. The analyst is for example interested in exploring the general regions of dangerous set plays and how the set plays were executed in the different regions. Computing and providing a valuable overview visualization is quite challenging as there exists high overlap of player trajectories. We propose several visualizations with different levels of aggregation to foster different views to the data suited to the analyst's needs.

\subsection{Dangerousness Measure}

The key to effective overview visualization is to derive and visualize domain-specific measures of interest from the data. In our case, the notion of dangerousness comes to mind when analyzing soccer situations. Visualizing the dangerousness of a set play is an effective way to get an impression of the success of a team's set play variations. Furthermore, visualizing the dangerousness by visual displays that guide the attention of the analyst to important parts in the data can support the interactive exploration process. The dangerousness of a set play depends on several parameters. We compute the measure by taking the type of free kick (e.g., pass, cross, shot, etc.), distance to the goal, shooting power as well as the outcome of the set play (e.g., shot on goal) into

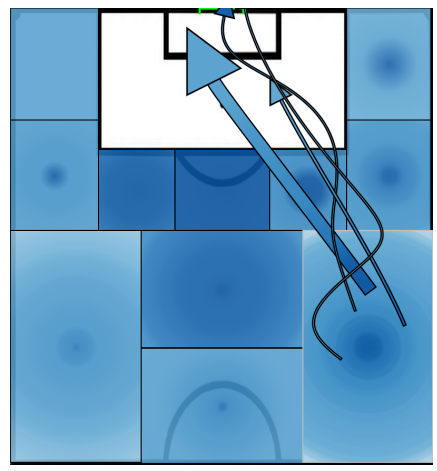

Figure 2. The analyst can display generalized free kicks by a mouse over. The thickness indicates the frequency of free kicks that were executed this way.

account. The calculation comprises a weighted sum of these parameters with subsequent normalization to the range $[0,1]$. We experimented with different normalization techniques (e.g. min-max-normalization) but found log-normalization to yield the best results. Especially robustness with respect to large dangerousness disparities and visualization interpretability were superior with log-normalization. The mapping to color is performed linearly per default to the range of white to blue (see Figure 3) according to the normalized value. We additionally offer the possibility to define own color mappings.

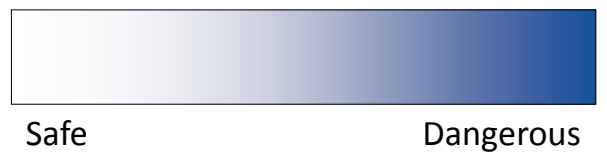

Figure 3. The color map used to distinguish dangerous set plays (blue) and safe set plays (white).

\subsection{Different Levels of Aggregation}

Depending on the overall analysis task different levels of aggregation are needed for the overview visualization. We provide four types of overview visualizations, as displayed in Figure 1 and Figure 2. On the highest aggregation level, we spatially aggregate the data into regions as displayed in Figure 1 (a). The regions were defined by a soccer expert reflecting a semantically meaningful partition of the soccer pitch. Our expert has been an active soccer player for 24 years and has been working as a coach for 10 years; he is currently employed by the German soccer club FC Bayern München. The soccer expert defined the partitions as regions in which different kind of tactical behavior is expected. The regions are colored according to the computed dangerousness of the free kicks within the respective region. For more detailed views of the free kicks, we provide a combination of regions and voronoi cells (see Figure 1 (b)). The reason we use a voronoi separation is to subdivide the soccer pitch into distinguishable areas according to set play execution. Given a set of points, the voronoi separation is calculated by assigning 
subregions to these points. The sides of these regions are obtained by dividing nearby points by a line. More details can be visualized by voronoi cells being not spatially aggregated depicted in Figure 1 (c). In these views, the analyst is able to get an impression of each individual free kick and its dangerousness. Furthermore, we provide possibilities to further drill-down into the underlaying movement data. As shown in Figure 2, we display aggregated trajectories via mouse hovering to indicate the movement and, consequently, tactical decisions.

\subsection{Importance-Driven Distortion}

The provided voronoi set play visualizations enables the analyst to inspect each captured free kick and to guide the analysis process. The challenge of voronoi cells is however that dense areas with many set plays will suffer from resulting small voronoi cells and that the distribution of cell shape and size may be irregular, depending on the data. An example can be seen in Figure 1 (b) and (c), where there are far more free kicks on the bottom right than, for example, on the bottom left.

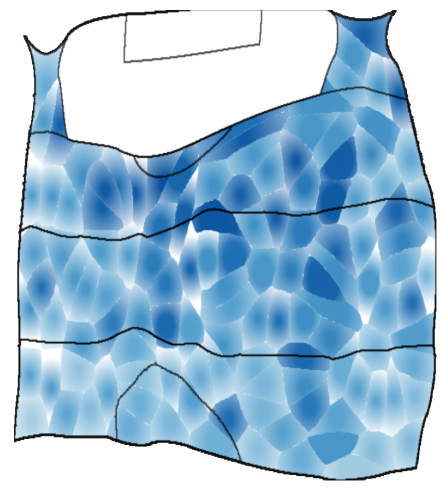

Figure 4. Distorted view of the soccer pitch following a cartogram-like visualization approach ensuring that each voronoi cell has the same area.

To tackle the problem of diverse cell size, we designed a visualization that ensures that every voronoi cell has approximately the same area and, therefore, distorts the original soccer pitch. This cartogram-like visualization [19] can exemplary be seen in Figure 4. The distortion clearly communicates the focus of free kicks on the right wing. In order to compute these cartogram-like visualizations, we use parts of ScapeToad (http://scapetoad.choros.ch/) and a diffusion-based algorithm [20] designed by Gastner and Newman.

\section{Zoom and Filter}

In Visual Analytics, overviews are accompanied and enhanced by zooming and filtering capabilities. Consequently, we provide the possibility to focus on more specific and detailed data aspects. This includes that coaches are interested in understanding the way opposing teams play their set plays, which formation they use, which player will take which role and where the ball is going to be played.

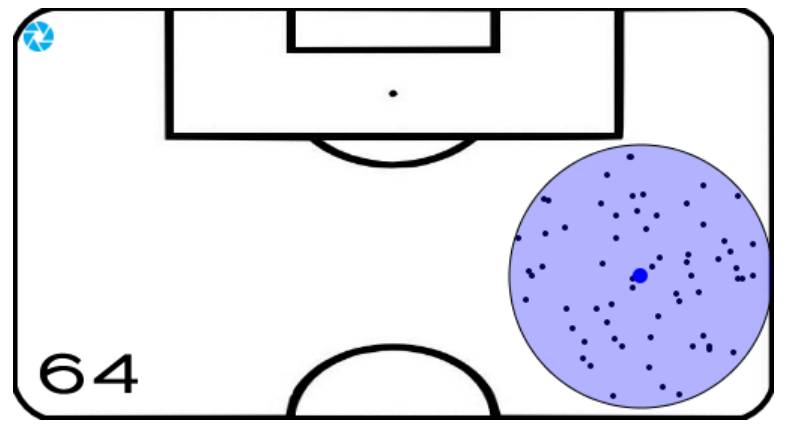

Figure 5. Starting positions of selected free kicks. The resulting formation prediction can be seen in Figure 6 (a).

\subsection{Formation Analysis}

When preparing for new opponents, coaches want to predict how a team is going to position itself during a set play. Therefore, we need to visualize the most probable position of each player as well as develop a visual feedback to indicate the uncertainty of our prediction for specific players. Starting the formation analysis, the user selects for which free kick position a prediction shall be performed (displayed by the blue dot in Figure 5). Black dots represent starting positions of recorded set plays around the selected position which means that the positioning and movement behavior of this set plays is taken into account.

The prediction is performed based on the selected position and historical data. We separately cluster the locations of each player at the beginning of free kicks close to the selected position in order to overcome outlier and noise influences. The centroid of the largest cluster will be the predicted position of the respective player. Each player is visually represented by a red jersey symbol with his starting number. The transparency of the jersey symbol indicates the uncertainty of the prediction as depicted in Figure 6. The calculation of the probability of the location of player $X$ is shown in Equation 1. We define a minimal probability of 0.2 to ensure visibility of the players, even if their positions are extremely uncertain.

$$
\mathbf{P}(X)=\max \left(0.2, \frac{\mid \text { largest cluster of } X \mid}{\mid \text { considered set plays } \mid}\right)
$$

We do not display all players of one team, but show the players with respect to their formal position (e.g., a team only has a specific amount of defenders, midfielders) and their probability to be in the line-up based on their number of matches. If a certain player displayed on the pitch is injured, the analyst can interactively remove that player and chose a replacement. To further indicate tactical behavior, we introduce a heat map visualization for set plays, as shown in Figure 7. The motivation of a heat map visualization is to show the most frequent execution paths. The darker the area is drawn, the more often the ball passed this area. Aggregating the data like this supports the detection of patterns as well as anomalies and serves as a way to gain an 


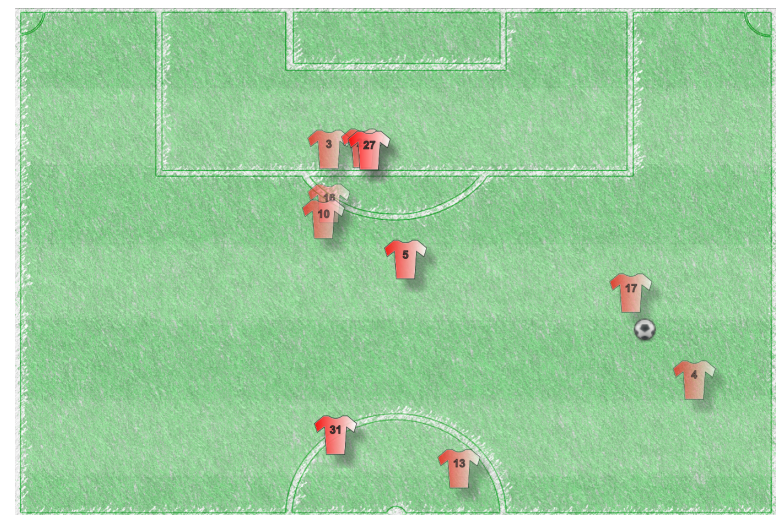

(a) Free Kick from the right wing

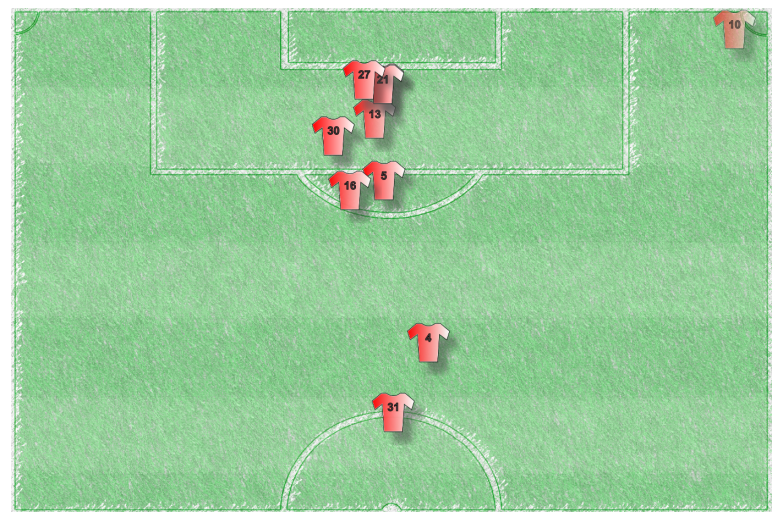

(b) Corner from the right wing

Figure 6. The formation prediction displays the team's positioning with the highest probability.

overview about the possible variations potentially employed in a match.

The uncertainty of a player in the predicted formation can be examined thoroughly as displayed in Figure 8. For a selected player, we visualize all of her or his positions at the moment of the set play. Player movement before (red line) as well as after (green line) the free kick are visualized. A pink line shows that the player received the ball in this set play. The dark clouds represent found positioning clusters for this player. Additionally, all player positions are connected by calculating their minimum spanning tree. Minimum spanning trees are often used as a means of clustering method (e.g., single linkage cluster analysis [21]). Our intention of using minimum spanning trees is to further enhance the visual notion of interconnectivity of the players' positions.

\subsection{Analysis of Tactic Variances}

The tactic variation view enables the analyst to dig deeper into the general approaches a team has in set play situations corresponding to the spatial context. We segment the data according to the path and stations of the ball during a set play. In order to achieve this segmentation, we cluster each set play based on its main events (cross, pass, shot, running with ball) with the TRACLUS-algorithm [22]. Afterwards,

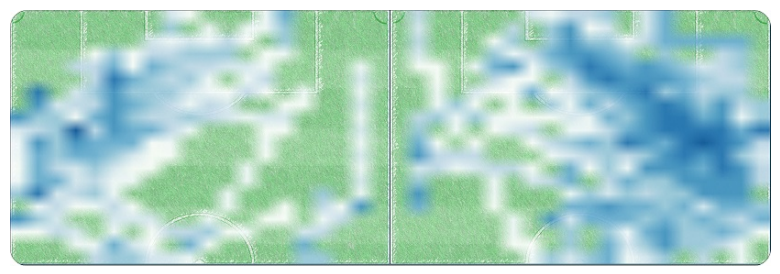

(a) Free Kick Heatmap

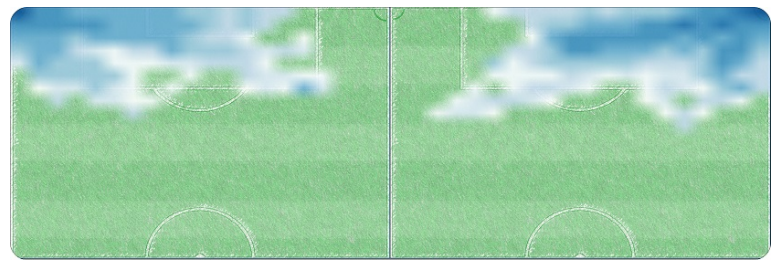

(b) Corner Heatmap

Figure 7. Heat map showing the most frequent set play execution paths. The left visualizations depict set plays from the left side and right visualizations represent set plays from the right side.

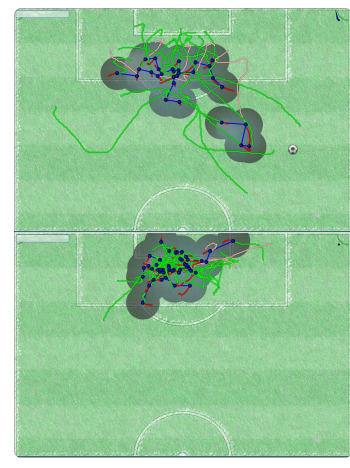

(a) Player 27

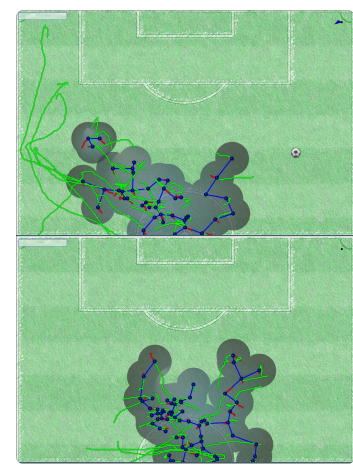

(b) Player 31
Figure 8. Player positioning view giving detailed information about a specific player (top shows free kicks and bottom depicts corners).

the arising clusters are grouped by the number and kind of events. The resulting clusters have a similar trajectory as well as similar number and kind of events. The analyst can inspect all found variation clusters as displayed in Figure 9. Each cluster is represented by a rectangle in which the main events are visualized by color. The size of a rectangle serves in a preattentive way to indicate how many set plays of the same variation style are in this cluster. It is given by the percentage of set plays falling into the variation the rectangle is presenting. The layout is realized by solving a packing problem for rectangular objects [23].

As the analyst decides to investigate a cluster further, we create a visualization showing the specific set play variant enriched by various symbols along the trajectory. Segments between two events are encoded by a describing symbol. Dribbling, for example, is encoded by a yellow waved line. The visualization of one single variation can be seen in Figure 10. We provide further means of filtering by a glyphbased filtering interface. The glyphs can show all different event possibilities within a cluster as shown in Figure 11. 


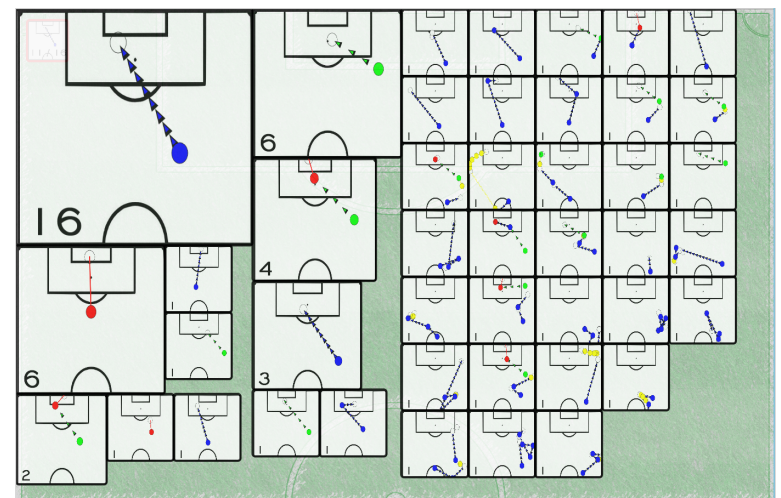

Figure 9. Cluster Variation Visualization for 75 free kicks from the middle to the right wing. Each cluster is represented by a rectangle. The size of each rectangle shows the amount of free kicks within.

For instance, filtering enables the user to look for direct goal shots or passes to other players. Furthermore, we derive generalized player movement by displaying abstracted player movement paths calculated by averaging the movement of each player in each set play of the variation cluster.

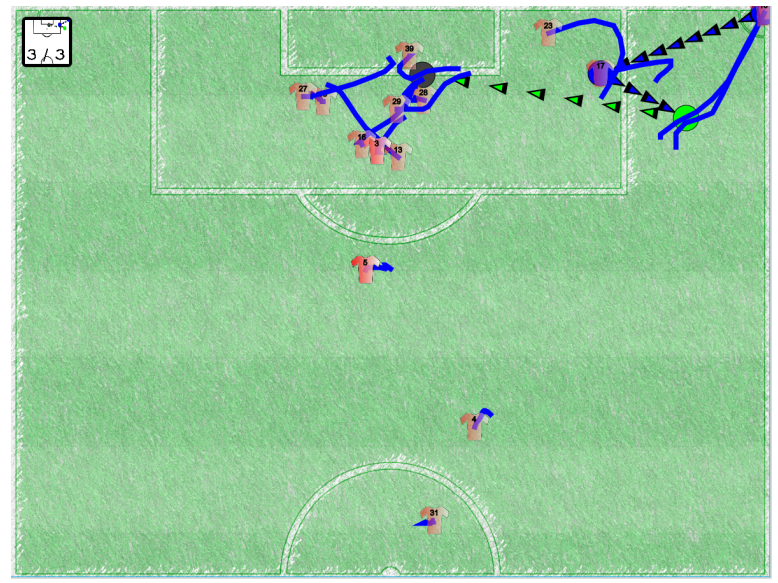

Figure 10. Identified corner variation in which player number 10 makes a short pass to player number 17 . He passes back to player 10 who makes a cross to the six-yard box.

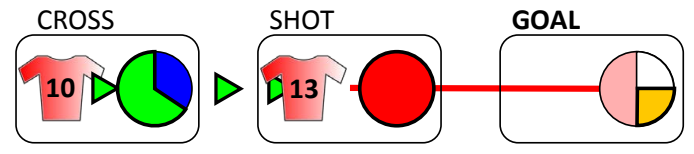

Figure 11. In this variation cluster, we filtered for all free kicks with crosses at the beginning.

\section{Details on Demand}

The verification and refining step during the analysis process is enabled by details on demand. Analysts may examine specific set play situations in detail and visually assess how these evolved. We therefore developed an animation in which analysts are able to animate the execution of a desired set play. We enriched this visual representation by adding various information such as player paths or blocked goal space by the so-called defensive wall in cases of a direct free kick. To compute the defensive wall for a free kick, we cluster the positions for players of the opposing team. Thereby, we only consider players who are positioned in the correct area that is the area between goal and free kick execution position, which according to play regulations is around $9.15 \mathrm{~m}$ from the starting point. From the resulting clusters, we choose the largest and if this cluster contains at least two players, we assign these players to the defensive wall. Additional meta-information (performer, distance, type, speed of the ball) is displayed via tooltips. The user can decide whether information on specific player types (e.g., defenders) are to be highlighted. Figure 12 shows an exemplary detail view comparing two free kicks. The attacking team is displayed by blue dots while the defending team is displayed by red dots. While paused, the overall ball trajectory and the starting positions of all players are being displayed. The exact movement of every actor gets animated in real-time otherwise. If desired, the analyst may add in additional contextual visualizations such as interaction spaces or free spaces that were developed in our previous work [12].

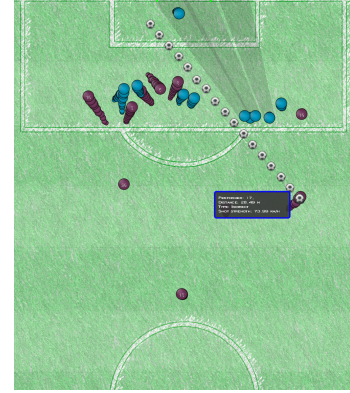

(a)

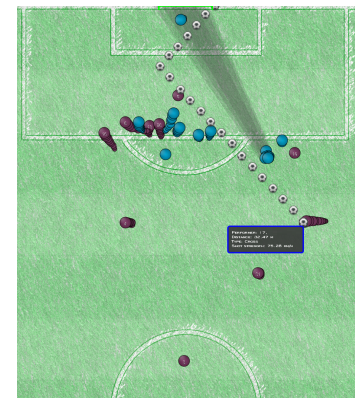

(b)
Figure 12. Detail view of two different free kicks with visualized defensive wall.

\section{Case studies}

In this section, we illustrate the usefulness of our proposed methods by analyzing real data. Therefore, we formulated three different analysis hypotheses:

- Can we identify predefined player roles during free kicks and corners and if so, how can they be described?

- Are there significant differences for free kicks and corners regarding player positions and roles?

- Which side is the preferred and stronger offensive side, and which side is more structured with respect to free kick and corner executions?

Each hypothesis is elaborated in a different case study and we describe how the methods and visualization presented in the previous sections can be employed. 


\subsection{Data}

The data used in our case studies was provided within a collaboration with the professional sport performance analyst Prozone (http://prozonesports.stats.com/). The dataset consists of 66 soccer matches with recorded spatial location data for each of the 22 soccer players with a temporal resolution of 100 milliseconds. Additionally, the data contains different manual annotated events like fouls, passes, and crosses. For each event a record consists of the type of event, position, timestamp, and event-specific information, such as the involved players. Since these events are manually annotated they are less frequent and accurate than the spatiotemporal movement data.

\subsection{Predefined Player Roles}

In our data we found evidence supporting the hypothesis that we can identify and describe predefined player roles during free kicks and corner kicks. Figure 6 (a) depicts the average position of players during 64 free kicks, whose execution positions are shown in Figure 5. During free kicks, five to six players are positioned around the penalty area, one player (number 5) is ready to receive the ball centrally and two players are more defensive, while the other two players are responsible for the execution of the free kick. In Figure 6 (b), we visualize the average position of the players during 86 corner kicks. There are seven players around the penalty area (with five players within), there are again two players for the defense and one player (number 10) performing the corners.

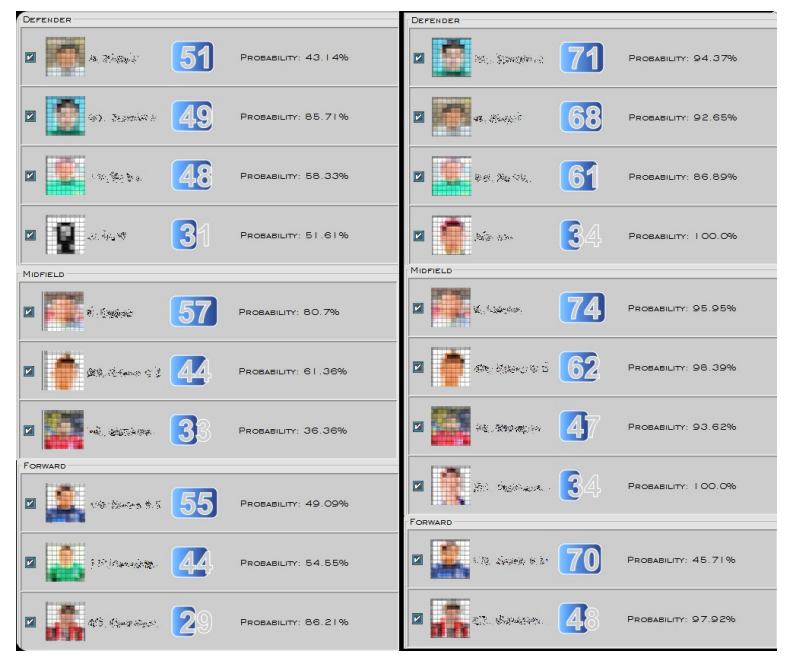

Figure 13. Probabilities of player positioning for free kicks (left) and corners (right).

Of special interest are the players 27 and 31 during the execution of free kicks and corners (see Figure 8 (a) and 8 (b), respectively). Player 27 is always positioned directly in front of the goal, indicating that this player is strong with headers. Player 31 never takes part offensively, but rather covers the own half around the middle line. These observations strongly indicate predefined roles of these players. Other interesting players are players 4 and 13. During corners, player 4 seems to support player 31 defensively, while during free kicks, he is involved in the execution. Player 13 instead is defensive during free kicks, when otherwise player 31 would be alone, and during corners, he plays rather offensively.

\subsection{Differences between Free Kicks and Corners}

Several commonalities are revealed in Figure 6 (a) and Figure 6 (b). Players 27 and 21, for example, are always closest to the opponent goal, while players 16 and 5 are directly behind players 27 and 21. Furthermore, player 31 is always performing defensive duties. If we compare the probabilities of the player positions of free kicks and corners (Figure 13), we can see that the positions at corners are almost perfectly predefined. Almost all players have a probability of over 90 percent, with the only exception being player 10 . This could also indicate the correlation of starting position and player positioning seeing corners as free kicks with a predefined execution position. The free kicks are selected in a larger area (see Figure 5) and therefore the probabilities shrink.

\subsection{Structure and Team Organization}

The preferred side of the considered team can roughly be estimated by comparing the obtained free kicks across the offensive side of the soccer pitch. In Figure 1 (b) and (c), we can clearly see a prevalence of free kicks on the right side of the pitch. About twice as much free kicks were obtained on the right side, compared to the left side. Also, the free kicks were overall more dangerous on the right side, indicated by the darker blue color of the respective voronoi cells. Another sign for a stronger offensive on the right side is the execution of the free kicks (and also corners), for which a heat map of the ball curves can be seen in Figure 7 (a) for free kicks and Figure 7 (b) for corners. The free kicks from the right side have a clearer path from execution position to the penalty area, compared to the free kicks from the left side, indicating more danger arising from free kicks executed from the right side. To a slightly lesser degree, this also holds true for corners (see Figure 7 (b)).

\section{Conclusion}

We presented a system following the visual informationseeking mantra of Ben Shneiderman to analyze a team's match plan. Therefore, we proposed appropriate analysis and visualization techniques for the different steps of "overview first" (Section 3), "zoom and filter" (Section 4), then "detailon-demand" (Section 5). We focused on match situations with mostly fixed parameters such as free kicks and corner kicks. In various use cases like the analysis of dangerousness or formation and variation prediction, we show that visual information seeking enables analysts to derive global strategies. However, there exists still a challenge to extract strategies 
from a set of observations. In the future, we plan to integrate a way to organize collections of findings and assemble them to form a bigger picture. Analysis management tools and analysis provenance tools can help to organize a strategy from sets of findings.

Interactive searching to navigate through the data is a crucial part of our system. We integrated methods for spatial searching (define start/end regions or sketches) but we may also need to search for similar player behavior on more abstract levels. How players adapt their behavior according to the interdependencies with other players from the own and the other team may, for example, be a very interesting analysis task. Our high level goal is to define a "behavior" feature vector.

Ultimately, a team's strategy and tactical behavior is not static over time, but adapts to specific (game-changing) events. We need methods to visualize these changes over time. As a simple approach we may show the temporal measurements as time series. It is, however, unclear how we can visualize the overall strategy or tactic. Semantic visualizations realized, for example, with the help of glyphs [24] may be an important first step with respect to this open problem.

\section{Acknowledgments}

The soccer data used in this publication were generously provided by Prozone.

\section{References}

[1] B. Travassos, K. Davids, D. Araújo, and P. T. Esteves, "Performance analysis in team sports: Advances from an ecological dynamics approach," International Journal of Performance Analysis in Sport, vol. 13, no. 1, pp. 83-95, 2013.

[2] B. Gonçalves, R. Marcelino, L. Torres-Ronda, C. Torrents, and J. Sampaio, "Effects of emphasising opposition and cooperation on collective movement behaviour during football small-sided games," Journal of sports sciences, vol. 34, no. 14, pp. 1346-1354, 2016.

[3] D. Keim, G. Andrienko, J.-D. Fekete, C. Görg, J. Kohlhammer, and G. Melançon, "Visual analytics: Definition, process, and challenges," in Information visualization. Springer, 2008, pp. 154-175.

[4] B. Shneiderman, "The eyes have it: a task by data type taxonomy for information visualizations," in Visual Languages, 1996. Proceedings., IEEE Symposium on, Sep 1996, pp. 336-343.

[5] M. Mitrotasios and V. Armatas, "Analysis of goal scoring patterns in the 2012 european football championship," United States Sports Academy, 2014.

[6] P. A. Legg, D. H. S. Chung, M. L. Parry, M. W. Jones, R. Long, I. W. Griffiths, and M. Chen, "Matchpad: Interactive glyph-based visualization for real-time sports performance analysis," Comput. Graph. Forum, vol. 31, no. 3, pp. 1255-1264, 2012. [Online]. Available: http://dx.doi.org/10.1111/j.1467-8659.2012.03118.x

[7] A. Lopes, S. Fonseca, R. Leser, and A. Baca, "Using voronoi diagrams to describe tactical behaviour in invasive team sports: an application in basketball," Cuadernos de Psicologia del Deporte, vol. 1, pp. 123-130, 2015.

[8] R. Basole, E. Clarkson, A. Cox, C. Healey, J. Stasko, and C. S. (Organizers), "First IEEE vis workshop on sports data visualization," Oct. 14, 2013.
[9] C. Perin, R. Vuillemot, and J.-D. Fekete, "Soccerstories: A kick-off for visual soccer analysis," Visualization and Computer Graphics, IEEE Transactions on, vol. 19, no. 12, pp. 2506-2515, 2013.

[10] M. Horton, J. Gudmundsson, S. Chawla, and J. Estephan, "Automated classification of passing in football," in PAKDD (2), ser. Lecture Notes in Computer Science, T. Cao, E.-P. Lim, Z.-H. Zhou, T.-B. Ho, D. W.-L. Cheung, and H. Motoda, Eds., vol. 9078. Springer, 2015, pp. 319-330. [Online]. Available: http://dx.doi.org/10.1007/978-3-319-18032-8

[11] H. Janetzko, D. Sacha, M. Stein, T. Schreck, D. Keim, and O. Deussen, "Feature-driven visual analytics of soccer data," in Proc. IEEE Conference on Visual Analytics Science and Technology, 2014, pp. 13-22, peer-reviewed full paper.

[12] M. Stein, H. Janetzko, T. Breitkreutz, D. Seebacher, T. Schreck, M. Grossniklaus, I. Couzin, and D. Keim, "Director's Cut: Analysis and Annotation of Soccer Matches," IEEE Computer Graphics and Applications (CG\&A), 2016, special Issue Sports Data Visualization. Accepted for publicaton.

[13] J. Perl, A. Grunz, and D. Memmert, "Tactics analysis in soccer-an advanced approach," International Journal of Computer Science in Sport, vol. 12, no. 1, pp. 33-44, 2013.

[14] L. Gyarmati and X. Anguera, "Automatic extraction of the passing strategies of soccer teams," CoRR, vol. abs/1508.02171, 2015. [Online]. Available: http://arxiv.org/abs/1508.02171

[15] R. Bartlett, C. Button, M. Robins, A. Dutt-Mazumder, and G. Kennedy, "Analysing team coordination patterns from player movement trajectories in soccer: methodological considerations," International Journal of Performance Analysis in Sport, vol. 12, no. 2, pp. 398-424, 2012.

[16] P. A. Legg, D. H. Chung, M. L. Parry, M. W. Jones, R. Long, I. W. Griffiths, and M. Chen, "Matchpad: Interactive glyph-based visualization for real-time sports performance analysis," in Computer Graphics Forum, vol. 31, no. 3pt4. Wiley Online Library, 2012, pp. 1255-1264.

[17] H. Pileggi, C. D. Stolper, J. M. Boyle, and J. T. Stasko, "Snapshot: Visualization to propel ice hockey analytics," Visualization and Computer Graphics, IEEE Transactions on, vol. 18, no. 12, pp. 28192828, 2012.

[18] T. Polk, J. Yang, Y. Hu, and Y. Zhao, "Tennivis: Visualization for tennis match analysis," IEEE Trans. Vis. Comput. Graph., vol. 20, no. 12, pp. 2339-2348, 2014. [Online]. Available: http://dx.doi.org/10.1109/TVCG.2014.2346445

[19] W. Tobler, "Thirty five years of computer cartograms," ANNALS of the Association of American Geographers, vol. 94, no. 1, pp. 58-73, 2004.

[20] M. T. Gastner and M. E. Newman, "Diffusion-based method for producing density-equalizing maps," Proceedings of the National Academy of Sciences of the United States of America, vol. 101, no. 20, pp. 7499-7504, 2004.

[21] J. C. Gower and G. Ross, "Minimum spanning trees and single linkage cluster analysis," Applied statistics, pp. 54-64, 1969.

[22] J.-G. Lee, J. Han, and K.-Y. Whang, "Trajectory clustering: a partitionand-group framework," in Proceedings of the 2007 ACM SIGMOD international conference on Management of data. ACM, 2007, pp. 593-604.

[23] "Packing Lightmaps," http://www.blackpawn.com/texts/lightmaps/, accessed August 4, 2016.

[24] R. Borgo, J. Kehrer, D. H. Chung, E. Maguire, R. S. Laramee, H. Hauser, M. Ward, and M. Chen, "Glyph-based visualization: Foundations, design guidelines, techniques and applications," Eurographics State of the Art Reports, pp. 39-63, 2013. 